\title{
- El Sistema Nacional de Inversión Pública. Un Análisis Crítico
}

(1) Wal ter Andía V.

\section{RESUMEN}

El presente artículo explica algunas observaciones a los componentes del Sistema Nacional de Inversión Pública (SNIP), con el único propósito de contribuir a perfeccionar su implementación y lograr así sus objetivos propuestos.

Palabras Claves: Inversión Pública, Sistema Nacional de Inversión Pública. Sostenibilidad.

The Public InVestment National System. A CRitical Analysis ABSTRACT

The present article explains some comments on the components of the Public Investment National System (PINS), with the only purpose of contributing to perfecting its implementation, thus reaching its intended aim.

Key Words: Public Investment. Public Investment National System. Sustainability.

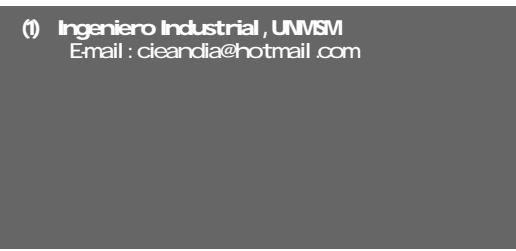

\section{INTRODUCCIÓN}

El Sistema Nacional de Inversión Pública (SNIP) se implementa en nuestro país con la finalidad de elevar la calidad de las inversiones y mejorar los niveles de equidad, eficiencia y sostenibilidad del gasto público, para ello, se determinó como unidad básica de análisis al proyecto y su ciclo de vida.

La incorporación del Sistema Nacional de Inversión Pública en nuestro medio significa un avance en materia de evaluación y toma de decisión racional de los proyectos propuestos por el sector público, ya que uniformiza conceptos, criterios y metodologías, además de brindar un marco legal que norma su operación.

Su aplicación en forma progresiva en las entidades del sector público fue un acierto metodológico pero con algunas limitaciones de información y poca flexibilidad en el uso de los manuales.

Es indudable que los avances son importantes, pero existen algunos componentes que no facilitan su aplicación, por lo tanto, es necesario mejorarlos. Las observaciones siguientes se proponen bajo una perspectiva critica, para que sirvan de reflexión de todas aquellas personas que, en su quehacer diario llevan consigo las inquietudes por comprender y dar respuestas a interrogantes en esta materia.

\section{ANTECEDENTES DEL SNIP}

El referente inmediato anterior al Sistema Nacional de Inversión Pública del Perú lo constituye el Sistema Nacional de Planificación, el mismo que fue desactivado al disolverse su órgano rector el Instituto Nacional de Planificación el año de 1992, asumiendo dichas funciones el Ministerio de Economía y Finanzas.

Es así que el año 2000, se crea el Sistema Nacional de Inversión Pública del Perú a través de la Ley № 27293, con la finalidad de optimizar el uso de los recursos públicos destinados a la inversión y buscando lograr los siguientes objetivos: la aplicación del Ciclo de Proyectos, fortalecer la capacidad de planeación del sector público y crear las condiciones para la elaboración de los planes de inversión multianuales.

El Sistema Nacional de Inversión Pública está conformado por:

1. La Dirección General de Programación Multianual del Sector Públi- 

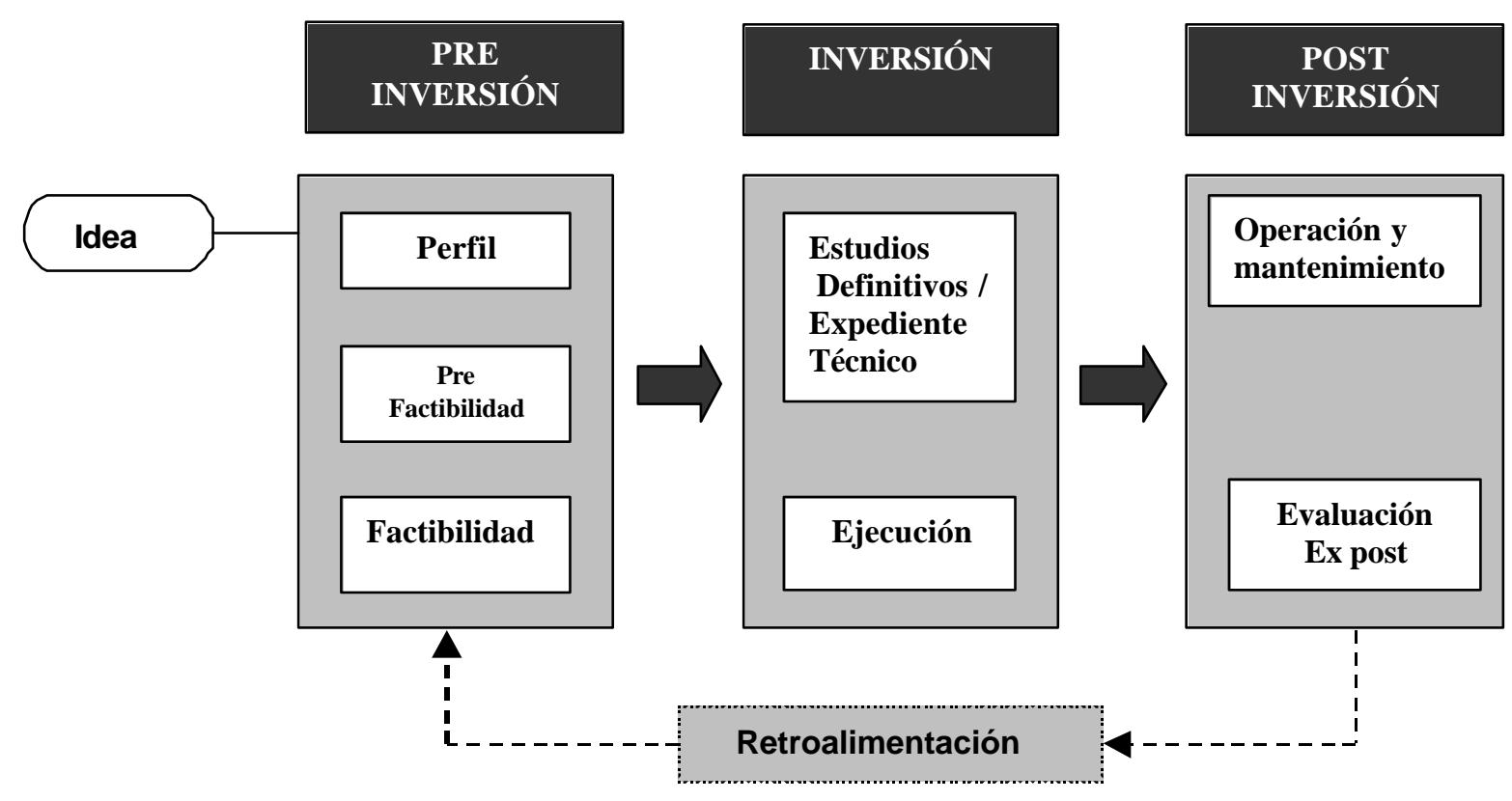

Figur a 1. Fases de un proyectode inversión pública

co (DGPM) del Ministerio de Economía y Finanzas, es la más alta autoridad técnica normativa del Sistema.

2. Los Órganos Resolutivos, es la máxima autoridad ejecutiva de cada sector.

3. Las Oficinas de Programación e Inversiones (OPI), es el órgano técnico del Sistema en cada sector.

4. Las Unidades Formuladoras (UF), son las dependencias o entidades del sector público registradas ante la DGPM, es la encargada de elaborar los estudios de pre inversión.

5. Las Unidades Ejecutoras (UE), son las dependencias o entidades del sector público con capacidad legal para ejecutar proyectos de inversión pública.

La aplicación del Sistema Nacional de Inversión Pública fue en forma progresiva ya que en sus inicios excluía a los gobiernos locales de su aplicación, para posteriormente a través de la Resolución Directoral № 007-2003-EF/68.01, aprobar la Directiva № 0042003-EF/68.01 Directiva para los Gobiernos Regionales y Gobiernos locales donde se determina incluirlos según parámetros establecidos.

Los proyectos de inversión pública se sujetan a las siguientes fases:

a. Fase de Preinversión: se realizan los estudios para sustentar y obtener la viabilidad del proyecto.

b. Fase de Inversión: se inicia una vez obtenido la viabilidad del proyecto.

c. Fase de Post Inversión.

\section{CONSIDERACIONES AL SNIP}

Las observaciones que se plantean se refieren a los distintos elementos del Sistema y se desarrollan en función a su impacto:

Primero, para que el SNIP cumpla con sus objetivos, es importante que se desarrolle una cultura de proyectos, ello requiere de la formación de profesionales con especialización en la materia. En nuestro medio se han realizado capacitaciones que por su estructura y la cantidad de horas, resultan insuficientes; es más, en el país, esta materia se desarrolla académicamente con muchas limitaciones.

Por la naturaleza de los cursos, siempre los temas de formulación y evaluación quedan pendientes de ser desarrollados, por lo que sería necesario programar cursos donde se expliquen temas específicos (evaluación social, oferta y demanda, etc.); también, es recomendable realizar capacitaciones según los niveles de conocimiento de los participantes.

Todo esto, influirá en mejorar la calidad de los proyectos y así facilitar el proceso de viabilidad así como de efectividad de las unidades formuladoras.

Segundo, en lo que respecta a los manuales para la preparación de los proyectos disponibles en el portal del Ministerio de Economía y Finanzas es necesario que la metodología empleada en estos documentos sea más práctica y consistente en la pre- 
sentación de casos, tales que, se mantenga la continuidad de ejemplos enlazados con la temática y relegar la rigurosidad de algunos tópicos que en la realidad son difíciles de aplicar por la complejidad del uso de información en nuestro medio. El aprendizaje es un proceso a desarrollar en etapas, por tanto, las cualidades de los documentos de consulta deben caracterizarse por su claridad, simplicidad y flexibilidad.

Tercero, el SNIP establece contenidos mínimos para la presentación de proyectos, específicamente a nivel de perfil contiene una estructura un poco rígida, por ejemplo, para proyectos de tipo productivos es necesario la incorporación de variables de marketing, es decir, el precio, distribución, descripción de las características del producto, etc. Es cierto que estamos analizando proyectos del sector público pero puede darse el caso, que se presenten estos tipos de proyectos, como por ejemplo, un centro preuniversitario de una universidad.

Asimismo, en el esquema citado los costos son considerados antes que los beneficios, siendo lo correcto primero la identificación de los beneficios ya que a partir de ello se hallan los costos.

Cuarto, la tasa de descuento es aquel parámetro que mide el uso de los recursos del estado y debe incorporar el riesgo del sector en donde se dirigirá el proyecto, el SNIP define una tasa del $14 \%$ para todos los proyectos del sector público; la interrogante sería, si un proyecto del sector agricultura tiene el mismo riesgo que un proyecto del sector educación, entonces ¿se debe usar la misma tasa de descuento?.

También, puede darse el caso que un proyecto tenga dos fuentes de financiamiento una de los recursos del estado y la otra mediante préstamo del sistema financiero, aquí la interrogante sería que tasa de descuento se debe emplear. Para dar respuesta se tiene que diferenciar los conceptos del flujo de caja económico y el financiero; en el primero encontramos la rentabilidad global de todo el proyecto, por tanto la tasa de descuento debe ser el promedio ponderado del $14 \%$ y del costo del financiamiento; en el flujo de caja financiero encontramos la rentabilidad por el aporte que realiza el estado, entonces, aquí la tasa es del $14 \%$.

Y quinto, el tipo de análisis utilizado en la evaluación de proyectos es preferentemente el costo beneficio; en muchos casos se debe utilizar otra metodología ya que los beneficios son difíciles de llevarlos a valores monetarios, el SNIP define al costo efectividad pero en muchos casos se utiliza el costo eficiencia. La diferencia se da en que el primero se relaciona los costos con algún indicador que represente a los beneficios, en el segundo caso, se relacionan los costos con la cantidad de usuarios.

\section{CONCLUSIONES}

Para obtener mejores resultados en la implementación del Sistema Nacional de Inversión Pública es necesario corregir las metodologías de transmisión de información hacia los usuarios, adecuar los parámetros y esquemas de presentación de los proyectos a las características propias de las necesidades de sus usuarios.

\section{BIBLIOGRAFÍA}

1. Andía Valencia, Walter. (2004) Gerencia de Proyectos, El Saber Editores, Lima Perú.

2. Ministerio de Economía y Finanzas. (2003) Guía General de Identificación, Formulación y Evaluación Social de Proyectos de Inversión Pública. Lima Perú. 